\title{
Epidemiologia da Doença de Alzheimer em Sergipe no período de 2008 a 2018: morbidade hospitalar e mortalidade
}

\author{
Epidemiology of Alzheimer's disease in Sergipe from 2008 to 2018: hospital morbity and mortality \\ Epidemiología de la enfermedad de Alzheimer en Sergipe de 2008 a 2018: morbilidad hospitalaria y \\ mortalidad
}

Recebido: 29/03/2021 | Revisado: 07/04/2021 | Aceito: 08/04/2021 | Publicado: 19/04/2021

João Paulo Siqueira Correia

ORCID: https://orcid.org/0000-0002-8214-0853 Universidade Tiradentes, Brasil

E-mail: jpsiqueirabrr@gmail.com

André Luiz Baião Campos

ORCID: https://orcid.org/0000-0002-5794-7196 Universidade Tiradentes, Brasil

E-mail: andre.baiao@souunit.com.br

Samara Siqueira Correia

ORCID: https://orcid.org/0000-0003-0191-9572 Centro Universitário Estácio de Sergipe, Brasil

E-mail: siqueira.correia@gmail.com

Crysvânia Santos Nascimento

ORCID: https://orcid.org/0000-0001-5760-4911

Universidade Tiradentes, Brasil

E-mail: crysvania39@gmail.com

\begin{abstract}
Resumo
A Doença de Alzheimer é a doença neurodegenerativa mais comum e principal causa de demência em todo o mundo, sendo um dos grandes desafios da saúde no século XXI. Nos últimos anos, a demência representou a quinta principal causa de morte no mundo, com cerca de 2,4 milhões de óbitos. Este trabalho teve como objetivo analisar os dados epidemiológicos da morbidade hospitalar e a mortalidade ocasionadas pela Doença de Alzheimer no Estado de Sergipe, entre os anos de 2008 e 2018. Trata-se de um estudo epidemiológico descritivo, retrospectivo e transversal realizado com dados do DATASUS. No período analisado, foram registradas apenas 21 internações, sendo treze do sexo feminino, doze atendimentos foram feitos em pacientes com 80 anos ou mais. Dados como raça/cor foram ignorados em 18 pacientes atendidos. Com relação a mortalidade, foram registrados 979 óbitos. A maioria dos moradores, 547, eram residentes de Aracaju. O sexo feminino foi o mais acometido com 645 casos e a faixa etária mais acometida foi a de pacientes com 80 anos ou mais, com 697 casos. Através do estudo foi possível avaliar a falta alimentação do sistema, principalmente no Sistema de Informações Hospitalares, uma vez que há poucos casos para serem analisados e algumas informações não são adequadamente registradas. É preciso ressaltar também a importância e contribuição de bases de dados como o DATASUS, pois a disponibilidade de informações públicas é essencial para execução de medidas de ação em saúde, realizadas de acordo com as características epidemiológicas das enfermidades estudadas.
\end{abstract}

Palavras-chave: Doença de Alzheimer; Epidemiologia; Morbidade hospitalar; Mortalidade; Sergipe.

\begin{abstract}
Alzheimer's disease is the most common neurodegenerative disease and the main cause of dementia worldwide, being one of the major health challenges in the 21st century. In recent years, dementia has represented the fifth leading cause of death in the world, with about 2.4 million deaths. This study aimed to analyze the epidemiological data on hospital morbidity and mortality caused by Alzheimer's Disease in the State of Sergipe, between the years 2008 and 2018. This is a descriptive, retrospective and cross-sectional epidemiological study conducted with data from DATASUS . In the analyzed period, only 21 hospitalizations were registered, thirteen of which were female, twelve visits were made to patients aged 80 years or older. Data such as race / color were ignored in 18 patients seen. Regarding mortality, 979 deaths were recorded. Most of the residents, 547, were residents of Aracaju. The female sex was the most affected with 645 cases and the most affected age group was that of patients aged 80 years or older, with 697 cases. Through the study it was possible to assess the lack of food in the system, mainly in the Hospital Information System, since there are few cases to be analyzed and some information is not properly registered. It is also necessary to emphasize the importance and contribution of databases such as DATASUS, since the availability of public information is essential for the implementation of health action measures, carried out according to the epidemiological characteristics of the studied diseases.
\end{abstract}

Keywords: Alzheimer's disease; Epidemiology; Hospital morbidity; Mortality; Sergipe. 


\begin{abstract}
Resumen
La enfermedad de Alzheimer es la enfermedad neurodegenerativa más común y la principal causa de demencia en todo el mundo, siendo uno de los mayores desafíos de salud en el siglo XXI. En los últimos años, la demencia ha representado la quinta causa principal de muerte en el mundo, con alrededor de 2,4 millones de muertes. Este estudio tuvo como objetivo analizar los datos epidemiológicos de la morbilidad y mortalidad hospitalaria por enfermedad de Alzheimer en el estado de Sergipe, entre los años 2008 y 2018. Se trata de un estudio epidemiológico descriptivo, retrospectivo y transversal realizado con datos de DATASUS. En el período analizado solo se registraron 21 hospitalizaciones, de las cuales trece fueron del sexo femenino, se realizaron doce visitas a pacientes de 80 años o más. Los datos como la raza / color se ignoraron en 18 pacientes atendidos. En cuanto a la mortalidad, se registraron 979 defunciones. La mayoría de los residentes, 547, eran residentes de Aracaju. El sexo femenino fue el más afectado con 645 casos y el grupo de edad más afectado fue el de pacientes de 80 años o más, con 697 casos. A través del estudio se pudo evaluar la falta de alimentos en el sistema, principalmente en el Sistema de Información Hospitalaria, ya que hay pocos casos para analizar y alguna información no está debidamente registrada. También es necesario enfatizar la importancia y el aporte de bases de datos como DATASUS, ya que la disponibilidad de información pública es fundamental para la implementación de las acciones de acción en salud, realizadas de acuerdo con las características epidemiológicas de las enfermedades estudiadas.
\end{abstract}

Palabras clave: Enfermedad de Alzheimer; Epidemiología; Morbilidad hospitalaria; Mortalidad; Sergipe.

\title{
1. Introdução
}

A Doença de Alzheimer (DA) é reconhecida atualmente como a doença neurodegenerativa mais comum do mundo, sendo também a principal causa de demência e um dos grandes desafios para saúde global no século XXI (Scheltens, et al., 2016; Webers, Heneka, \& Gleeson, 2019; Agnihotri \& Aruoma, 2020; Carneiro, Morais, \& Pereira, 2020; Hao et al., 2020; Pini et al., 2020; Souza et al., 2020; Zhou et al., 2020).

A DA é caracterizada por condições multifatoriais que levam a uma degeneração neuronal generalizada e perda sináptica que compromete principalmente o hipocampo e o córtex, provocando atrofia cerebral difusa (Scheltens, et al., 2016; Webers, Heneka, \& Gleeson, 2019; Agnihotri \& Aruoma, 2020; Andrews, Fulton-Howard, \& Goate, 2020; Carneiro, Morais \& Pereira, 2020; Christopher, 2020; Franzmeier et al., 2020; Gate et al., 2020; Karikari et al., 2020; Janelidze et al., 2020; Pini et al., 2020; Zhou et al., 2020). A patologia primária é descrita pela formação de placas amilóides extracelulares formadas por depósitos peptídicos de amilóide- $\beta$ (A $\beta$ ), derivados da clivagem da proteína precursora de amilóide (APP) e emaranhados neurofibrilares intracelulares (NFT) compostos por proteína tau (proteína associada a microtúbulos) (Webers, Heneka, \& Gleeson, 2019; Pais, et al., 2020).

É uma doença neurodegenerativa crônica, com incidência e prevalência que aumentam de acordo com a idade e que causa declínio progressivo da cognição, concomitante a um declínio funcional provocado pela morte de células nervosas e consequente perda de tecido cerebral, culminando em níveis crescentes de incapacidade Webers, Heneka \& Gleeson, 2019; Andrews, Fulton-Howard, \& Goate, 2020; Carneiro, Morais \& Pereira, 2020; Pini et al., 2020; Wen et al., 2020). A DA parece ser uma das doenças multifatoriais humanas com o mais alto nível de herdabilidade (70\%) - um nível semelhante ao observado para a esquizofrenia (80\%) e bem acima dos encontrados para diabetes (40\%) e doença de Parkinson (30\%) (Bellenguez, GrenierBoley, \& Lambert, 2020).

Nos últimos anos, em parte pelo crescimento da população idosa globalmente, houve um aumento na incidência de doenças cerebrais em todo o mundo. As doenças neurodegenerativas representam uma grande causa de incapacidade funcional e com custos crescentes para a saúde pública mundial (Agnihotri \& Aruoma, 2020; Jia et al., 2020; Pais et al., 2020). O custo estimado para os cuidados com as demências, somente nos Estados Unidos, no ano de 2019, foi cerca de US\$290 bilhões de dólares, com previsão de aumento para até US\$1,1 trilhão de dólares em 2050 (Suryadevara, Klüppel, Monte, \& Willis, 2020).

Dados mundiais retratam que aproximadamente 40 milhões de pessoas, a maioria com mais de 60 anos, têm demência. A expectativa, segundo a Organização Mundial da Saúde (OMS), é que esse número deve triplicar até 2050, sendo reconhecida pela OMS como uma prioridade global de saúde pública (GBD 2016 Dementia Collaborators, 2019; Webers, Heneka \& Gleeson, 
2019; Carneiro, Morais \& Pereira, 2020; Karikari et al., 2020; Pais et al., 2020; Piovezan et al., 2020; Savulich, O’brien \& Sahakian, 2020; Santos, Bessa \& Xavier, 2020; Souza et al., 2020; Suryadevara et al., 2020).

No Brasil, vem ocorrendo uma transição demográfica e epidemiológica com mudança na pirâmide etária brasileira e envelhecimento populacional crescente. Prevê-se que a população com 60 anos ou mais atingirá 13,7\% do total em 2020, chegando a quase um quarto do total de habitantes do país em 2040 (Santos, Bessa \& Xavier, 2020). Em uma revisão de literatura de 2013 com dados entre 1995 a 2012, Burlá et al., estimaram a prevalência de demência no Brasil de 7,6\% em pessoas com 65 anos ou mais (Souza et al., 2020).

Em dezembro de 2013, o G8 declarou que a demência deveria tornar-se uma prioridade global e definiu a ambição de que a cura ou uma terapia modificadora da doença esteja disponível até 2025 (Scheltens, et al., 2016). De acordo com o estudo Global Burden of Disease do período de 1990 a 2016, a demência representou a quinta principal causa de morte no mundo, representando 2,4 milhões de mortes (Hao et al., 2020; Piovezan et al., 2020). Pessoas com demência têm um risco médio de mortalidade 2,6 vezes superior ao das pessoas na mesma faixa etária sem tal comorbidade (Piovezan et al., 2020). Há três classes de fatores de risco conhecidos para aumento da mortalidade na DA: características demográficas (idade avançada, sexo masculino); características clínicas da demência (gravidade, baixos escores no Mini-Mental, declínio cognitivo) e intervenções clínicas, principalmente medicamentos (indivíduos com demência podem ser mais sensíveis aos efeitos adversos dos medicamentos, incluindo mortalidade) (Barrett et al., 2020).

As Manifestações clínicas da Doença de Alzheimer são caracterizadas por perda inicial de memória de curto prazo seguida por déficits cognitivos graves subsequentes devidos à perda neuronal, com queda da capacidade cognitiva de raciocínio, acompanhadas de linguagem e distúrbios do movimento. (Webers, Heneka \& Gleeson, 2019).

O diagnóstico clínico de qualquer síndrome demencial depende de uma história do paciente e de seus cuidadores, testes neuropsicológicos e avaliação dos sintomas com o tempo (Scheltens et al., 2016). Um diagnóstico precoce é indispensável para retardar a progressão da enfermidade e reduzir o ônus da doença para familiares e o sistema de saúde, fornecendo tempo para o planejamento dos cuidados (Zendehbad et al., 2020). Hoje se utiliza de avaliações como histórico médico, teste de estado mental e técnicas de neuroimagem para confirmação do diagnóstico (Agnihotri \& Aruoma, 2020).

Atualmente, o campo do desenvolvimento de medicamentos referente à DA está direcionando seus esforços para o desenvolvimento de medicamentos modificadores de doença, sendo esses tratamentos mais propensos a ter sucesso quando administrados nos estágios iniciais da doença (Carneiro, Morais, \& Pereira, 2020).

As opções atuais de tratamento farmacêutico para a DA ainda estão limitadas a sintomas. O uso de intervenções com inibidores da acetilcolinesterase (IAChE) e a Memantina, um antagonista do receptor de N-metil-D-aspartato (NMDA), têm efeitos sintomáticos na cognição (Loureiro et al., 2020; Möllers et al., 2020). Além disso, em estudos sobre a doença, esses medicamentos também relataram benefícios significativos para a mortalidade e um atraso na ida de idosos para clínicas de repouso. No entanto, até agora, há poucos estudos epidemiológicos abrangentes investigando a associação entre o uso de medicamentos para a Doença de Alzheimer e hospitalizações (Möllers et al., 2020).

O objetivo do estudo foi avaliar o comportamento epidemiológico da mortalidade e morbidade hospitalar da Doença de Alzheimer no Estado de Sergipe entre os anos de 2008 e 2018 a fim de caracterizar o perfil epidemiológico da doença no Estado, baseado em dados secundários obtidos através do Sistema de Informações Hospitalares do Sistema Único de Saúde (SIH/SUS) e o Sistema de Informações sobre Mortalidade (SIM), ambos disponibilizados pelo Departamento de Informática do Sistema Único de Saúde do Brasil (DATASUS) através da plataforma Informações de Saúde (TABNET). 


\section{Materiais e Métodos}

Trata-se de um estudo epidemiológico descritivo, retrospectivo e transversal, baseado em dados secundários do Estado de Sergipe, obtidos através do Sistema de Informações Hospitalares do Sistema Único de Saúde (SIH/SUS) e o Sistema de Informações sobre Mortalidade (SIM), ambos disponibilizados pelo Departamento de Informática do Sistema Único de Saúde do Brasil (DATASUS) através da plataforma Informações de Saúde (TABNET).

O estudo epidemiológico descritivo tem por objetivo determinar a distribuição de doenças ou condições relacionadas à saúde, segundo o tempo, lugar e/ou características dos indivíduos e examina como a incidência ou a prevalência de uma doença ou condição relacionada à saúde varia de acordo com determinadas características, como sexo, idade, escolaridade e renda, outros (Lima-Costa \& Barreto, 2003).

De acordo com Gil (2002), o estudo retrospectivo é elaborado com base em registros do passado com seguimento até o presente. Só se torna viável quando se dispõe de arquivos com protocolos completos e organizados.

Já o estudo transversal, apresenta-se como uma fotografia ou corte instantâneo que se faz numa população por meio de uma amostragem, examinando-se nos integrantes da casuística ou amostra, a presença ou ausência da exposição e a presença ou ausência do efeito (ou doença), tendo como principais vantagens o fato de serem de baixo custo, e por praticamente não haver perdas de seguimento (Haddad, 2004 apud Hochman et al., 2005).

A pesquisa de abordagem quantitativa é capaz de identificar a natureza profunda das realidades, seu sistema de relações, sua estrutura dinâmica. Ela também pode determinar a força de associação ou correlação entre variáveis, a generalização e objetivação dos resultados através de uma mostra que faz inferência a uma população. Ao seu tempo também pode fazer inferências causais que explicam por que as coisas acontecem ou não de uma forma determinada (Esperón, 2017).

Foram selecionadas as informações relacionadas à morbidade hospitalar e mortalidade com base na $10^{\mathrm{a}}$ revisão da Classificação Internacional de Doenças (CID-10): G30 - Doença de Alzheimer. A escolha do período de 2008 a 2018 se deu pela disponibilidade em comum dos dados nos dois Sistemas de Informações através do DATASUS no momento da pesquisa, em novembro de 2020. As internações hospitalares e os dados sobre mortalidade foram analisados conforme ano de acometimento, município de residência, sexo, faixa etária, raça/cor, assim como regime de atendimento.

Nas informações sobre morbidade hospitalar também foi verificado o gasto total nos internamentos, caráter de atendimento e taxa de letalidade. Além desses dados, o SIM também disponibiliza para análise de dados o estado civil e escolaridade, ambos adicionados ao estudo.

Os dados adquiridos estão disponíveis na internet, disponibilizados pelo Ministério da Saúde para livre consulta, apresentando domínio público e preservando o sigilo dos seres humanos envolvidos, não necessitando da submissão ao Comitê de Ética em Pesquisa.

\section{Resultados e Discussão}

Segundo dados do último censo demográfico do Instituto Brasileiro de Geografia e Estatística (IBGE) realizado em 2010, Sergipe tem uma população total de 2.068.017 habitantes. Aracaju é o município mais populoso, com 571.149 pessoas e o município com o menor número de habitantes é Amparo de São Francisco, com 2.275 indivíduos. Cerca de 51,4\% da população é do sexo feminino: 1.062.976 de mulheres. A população idosa, com 60 anos ou mais, representa aproximadamente $9 \%$ do total de habitantes: 185.957 pessoas. Aracaju é a cidade com o maior número de idosos, 51.887 residentes.

Em Sergipe, no período de janeiro/2008 a dezembro/2018 a morbidade hospitalar do SUS/SE por Doença de Alzheimer foi de apenas 21 internações. A maior parte dos pacientes atendidos é de moradores da cidade de Lagarto - seis casos, seguido de Nossa Senhora de Socorro - quatro casos e Aracaju com apenas três internamentos. Aquidabã, Ilha das Flores, Itabaiana, 
Itabaianinha, Moita Bonita, Santa Luzia do Itanhy, Siriri e Tobias Barreto foram as outras cidades com dados de morbidade hospitalar pelo SUS/SE, com um caso oriundo de cada cidade.

É possível observar através do Gráfico 1 que a maior parte dos internamentos são de pessoas do sexo feminino (treze), contra (oito) do sexo masculino. A faixa etária mais atendida foi a de pacientes com 80 anos ou mais, com doze ocorrências. Entre os pacientes com 70 anos até 79 anos completos foram seis atendimentos. Em dois casos os pacientes tinham entre 60 e 69 anos. Foi atendido apenas um caso na faixa etária entre 50 e 59 anos e os pacientes com 80 anos ou mais, pela própria progressão da doença, são os que apresentam as maiores complicações na Doença de Alzheimer. Distúrbios musculares como prejuízo a deglutição levando a episódios de broncoaspiração e pneumonia assim como a insuficiência respiratória levam esses pacientes diagnosticados há mais anos a necessitar de auxílio cada vez mais recorrente para tratar complicações da DA ou até mesmo medidas preventivas a fim de diminuir a mortalidade.

Gráfico 1 - Internações hospitalares por sexo segundo faixa etária.

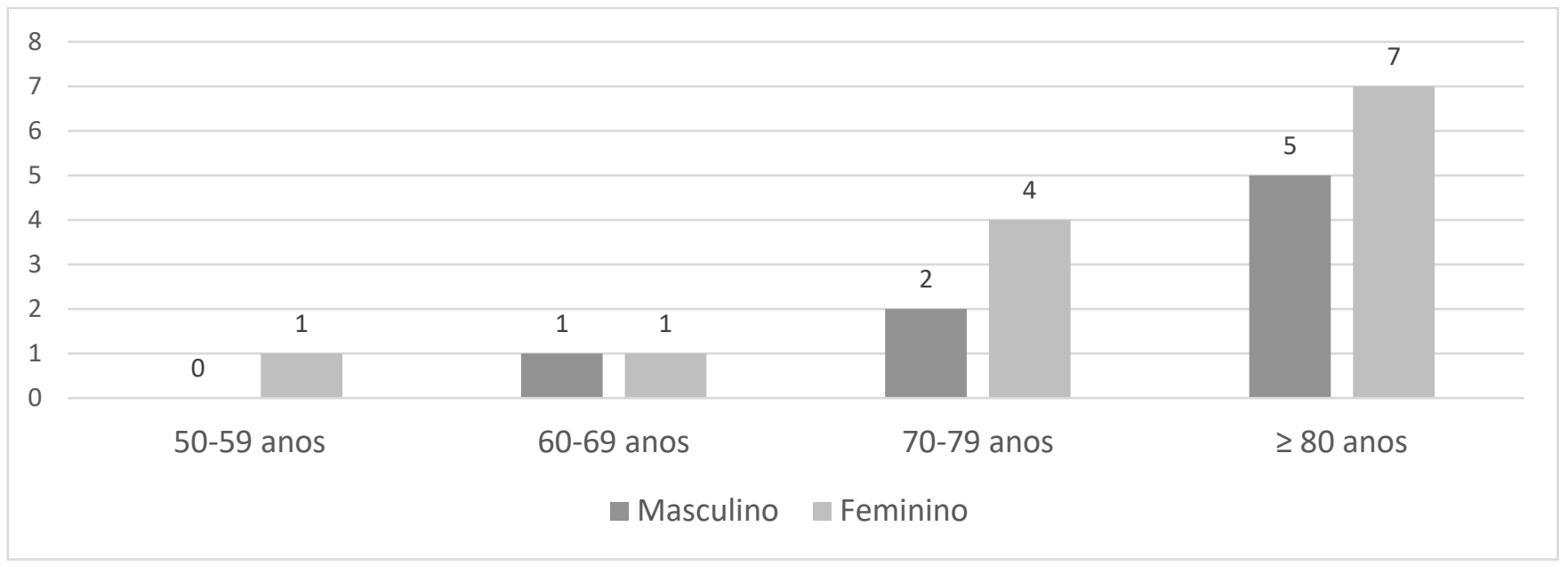

Fonte: Brasil. Ministério da Saúde. Sistema de Informações Hospitalares do SUS (SIH/SUS).

Houve um aumento progressivo nos atendimentos entre 2008 a 2017 e nova queda nos casos de internação no ano de 2018, com apenas um caso registrado. Os anos de 2008, 2011, 2012 e 2018 registraram apenas um caso em cada. O ano de 2014 apresentou dois casos. Em 2015, três casos. Já em 2016 foram fichados cinco casos. O maior número de casos foi registrado no ano de 2017, com sete ocorrências. Os anos de 2009, 2010 e 2013 não tiveram registros de casos, conforme descrito no Gráfico 2.

Gráfico 2 - Internações hospitalares segundo ano de atendimento.

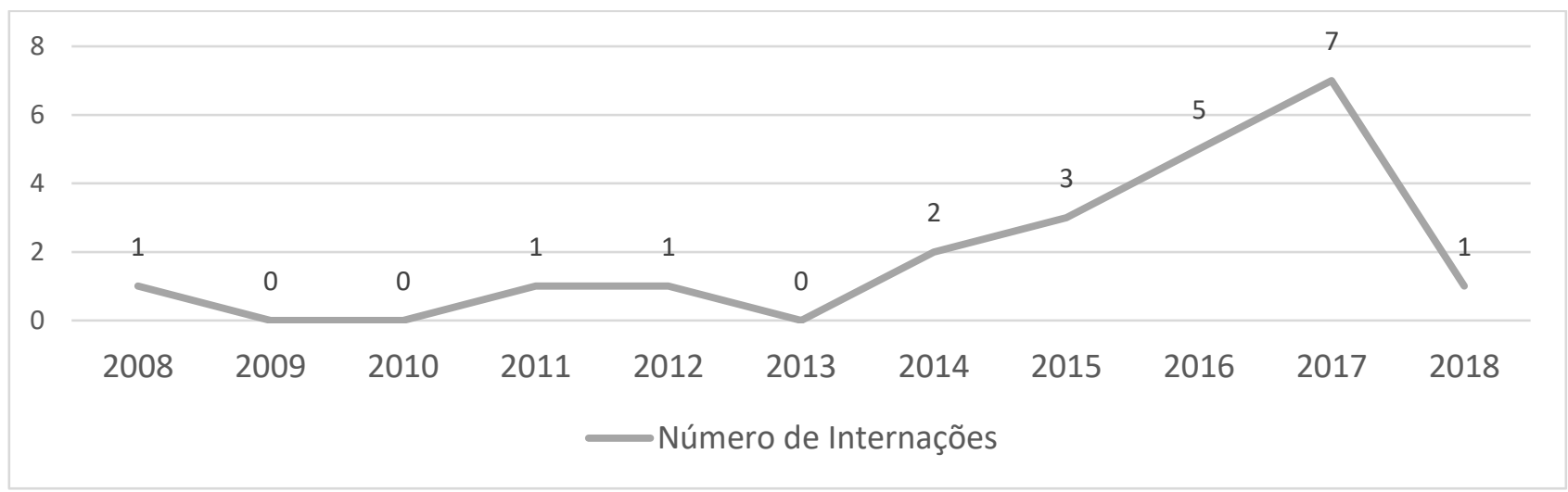

Fonte: Brasil. Ministério da Saúde. Sistema de Informações Hospitalares do SUS (SIH/SUS). 
A maior parte dos dados de cor/raça dos pacientes internados foi ignorada. Dezoito pacientes não tiveram a cor/raça detalhada. Dois pacientes eram da cor amarela e um pardo. Entre o regime de internamento, vinte casos foram atendidos em hospitais públicos e apenas um caso, na cidade de Estância foi atendido através de Entidade sem fins lucrativos. Vinte casos foram atendidos em caráter de urgência e apenas uma ocorrência foi registrada como atendimento eletivo.

A taxa de óbito foi de $23,8 \%$, com 5 óbitos registrados nesse período, sendo três pacientes do sexo masculino e dois do sexo feminino. Dois pacientes com idade entre 70 e 79 anos evoluíram a óbito, com uma taxa de letalidade de 33,33\%. Entre os 12 pacientes com 80 anos ou mais internados, dois morreram, letalidade de 16,66\%. O único paciente internado registrado com idade entre 50 e 59 anos faleceu, representando uma letalidade de $100 \%$.

O custo total de todas as vinte e uma internações foi de $\mathrm{R} \$ 16.426,56$ reais. Com os pacientes de 80 anos ou mais correspondendo a $86,94 \%$ do custo total, $\mathrm{R} \$ 14.282,15$ reais foram destinados apenas para essa faixa etária.

O estudo observou uma falta de alimentação ou até subnotificação dos quadros do Sistema de Informações Hospitalares do SUS (SIH/SUS), com apenas 21 internações por Doença de Alzheimer no período de dez anos avaliado. A DA como doença de base que propiciou a enfermidade da internação pode não ter sido referida, espelhando o número muito pequeno de internamentos no período analisado. Assim como a subnotificação dos internamentos em geral, a declaração de cor/raça dos pacientes também foi ignorado, com $85,71 \%$ dos casos sem raça definida.

Entre os anos de 2008 a 2018 foram registrados 979 óbitos no Estado de Sergipe segundo o Sistema de Informações sobre Mortalidade (SIM) do Ministério da Saúde. A maioria dos casos, 547 óbitos, foi registrada na cidade de Aracaju, representando 55,87\% dos registros. A segunda cidade com maior número de óbitos foi Itabaiana, com 54 casos e 5,51\% do total, seguida por Nossa Senhora de Socorro com 42 casos e Lagarto com 41 óbitos registrados.

No Gráfico 3, está ilustrado o aumento no número de óbitos no decorrer dos anos, com trinta e seis casos registrados em 2008 chegando a cento e quarenta e oito casos no ano de 2018. Do total de registros, 65,88\% foram óbitos de pacientes do sexo feminino, com 645 casos contra 334 casos do sexo masculino.

Gráfico 3 - Número total de óbitos por ano da ocorrência.

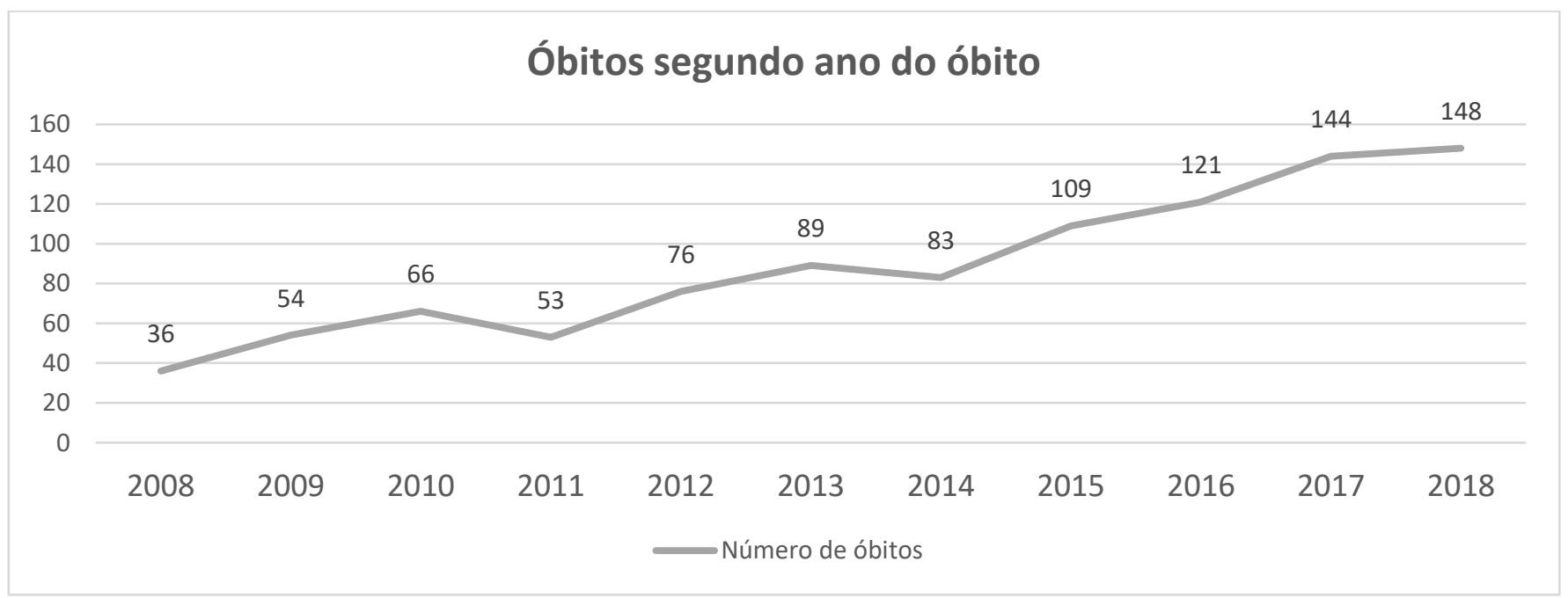

Fonte: Brasil. Ministério da Saúde. Sistema de Informações Hospitalares do SUS (SIH/SUS).

Ao visualizar o Gráfico 4 é possível notar que, no Brasil, o número de óbitos por Doença de Alzheimer é maior no sexo feminino, em contrapartida aos estudos que relatam o sexo masculino como um fator de risco para mortalidade na DA. O estudo realizado ratificou os resultados já encontrados em outros estudos sobre mortalidade da DA no país, o inverso do visto em outros estudos sobre mortalidade, uma vez que demonstrou que aproximadamente 2/3 dos óbitos relatados são do sexo feminino. Esses 
dados podem estar relacionados ao maior número de diagnóstico dado para o sexo feminino, uma vez que as mulheres tendem a buscar mais auxílio médico, sendo a DA, assim como outras patologias, subdiagnosticadas no sexo masculino. Essa taxa retrataria uma proporcionalidade no número de óbitos/mortalidade e o total de diagnósticos realizados e não uma relação do sexo feminino como fator de risco para aumento da mortalidade na Doença de Alzheimer.

A faixa etária mais acometida foi de pacientes com 80 anos ou mais, com 697 casos registrados dos 979 óbitos declarados, representando 71,19\% das mortes. A segunda faixa etária acometida foi entre 70 e 79 anos, com 226 casos registrados e 23,08\% do total. Do total de registros, 5,1\% foram registrados na faixa etária entre 60 e 69 anos, com 6 casos em menores de 60 anos, dentre esses, um registro em uma faixa etária extremamente incomum (25 a 29 anos), podendo ser um caso raro de Doença de Alzheimer hereditária bastante precoce ou erro de notificação. A paciente foi referida como do sexo feminino, branca, viúva, sem escolaridade, residente de Ribeirópolis e com óbito ocorrido em hospital.

Gráfico 4 - Óbitos por sexo segundo faixa etária.

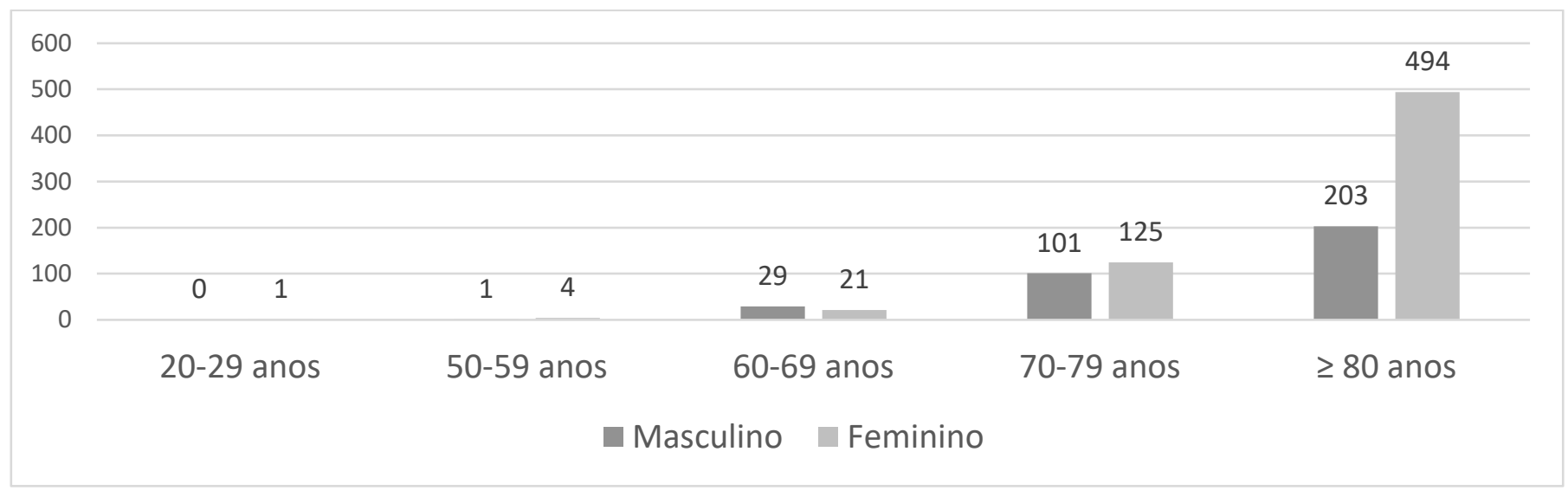

Fonte: Brasil. Ministério da Saúde. Sistema de Informações Hospitalares do SUS (SIH/SUS).

Esses dados ressaltam dois dos maiores fatores de risco para mortalidade na DA: doença avançada e progressão da doença. Os pacientes com 80 anos ou mais representam os que desenvolvem as maiores complicações ocasionadas pela neurodegeneração progressiva. Uma vez que a doença de Alzheimer surge, em sua maioria, em pacientes com mais de sessenta anos e a progressão da própria enfermidade leva a distúrbios musculares com prejuízo a deglutição levando a episódios de broncoaspiração e pneumonia e até desnutrição, podendo levar a ocorrência de insuficiência respiratória. Os pacientes diagnosticados há mais anos tendem a evoluir mais frequentemente com essas complicações e necessitam cada vez mais de tratamentos ou medidas preventivas a fim de diminuir a mortalidade.

Do total de 979 óbitos registrados, 437 pacientes eram viúvos, 44,63\% do total, 276 eram casados, 179 solteiros, 36 separados judicialmente e 51 tiveram outro estado civil ou situação civil ignorada no registro, de acordo com o descrito no Gráfico 5. 
Gráfico 5 - Óbitos segundo estado civil.

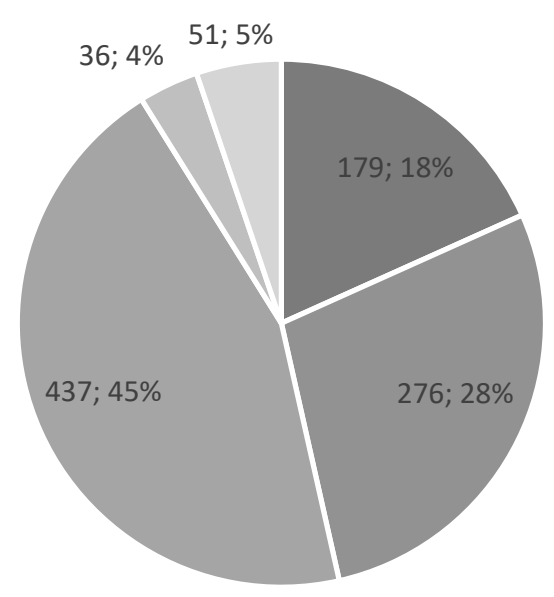

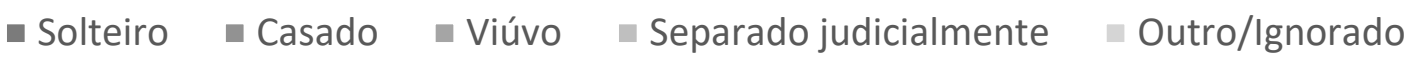

Fonte: Brasil. Ministério da Saúde. Sistema de Informações sobre Mortalidade (SIM).

Ao visualizar o Gráfico 6, com relação a cor e raça, 527 pacientes eram brancos, 53,83\% dos casos, 365 pardos ou $37,28 \%$ do total, 50 pretos, correspondente a 5,1\% dos registros. Apenas 02 pacientes foram declarados como cor amarela, $0,2 \%$ dos casos. Foram 3,5\% de óbitos com raça/cor ignorada, 35 casos. O papel da etnia/raça na mortalidade pela DA é inconclusivo, pois os estudos são discordantes sobre ser ou não um fator potencial de mortalidade e pode estar retratada uma proporcionalidade nos óbitos de acordo com a quantidade de diagnósticos totais realizados. Assim como o estado civil, que não parece afetar a mortalidade, mas um fator que acelera a ida a clínicas de repouso: viúvos e solteiros sem familiares próximos apresentam um retardo no diagnóstico e, por consequência, o início de tratamento, ocasionando uma progressão mais rápida dos sintomas.

Gráfico 6 - Óbitos segundo cor/raça.

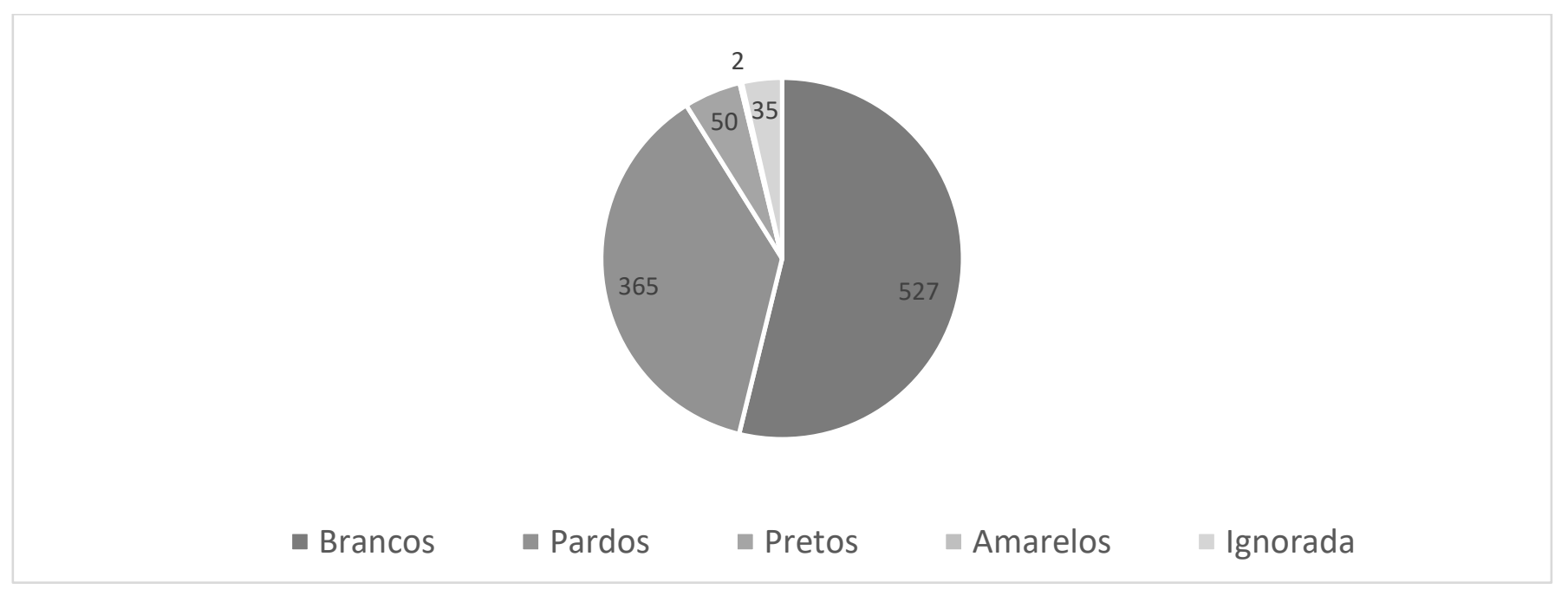

Fonte: Brasil. Ministério da Saúde. Sistema de Informações sobre Mortalidade (SIM).

A maioria dos casos registrados ocorrem em dois locais: hospital e o próprio domicílio. Os óbitos hospitalares representaram 50,76\% dos casos, com 497 registros, seguido pelos 470 óbitos ocorridos em domicílio, 48\% do total. Unidades 
de Pronto Atendimento (UPA) e outros estabelecimentos de saúde tiveram 04 óbitos, 02 casos ocorreram em via pública e 06 casos transcorreram em outros locais não especificados, de acordo com o detalhado no Gráfico 7.

Gráfico 7 - Óbitos por local de ocorrência.

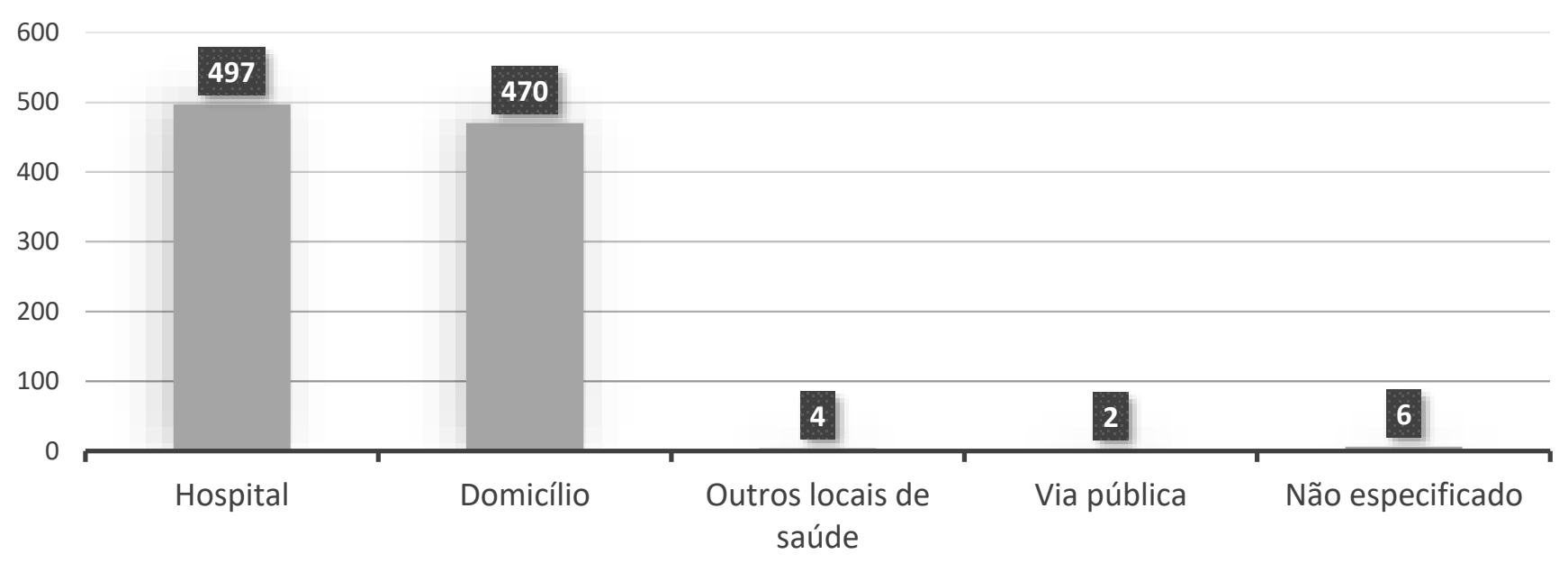

Fonte: Brasil. Ministério da Saúde. Sistema de Informações sobre Mortalidade (SIM).

No Gráfico 8 é possível visualizar um fator de proteção: a menor parte dos óbitos foi descrita na população com maior escolaridade. Os pacientes com 12 anos ou mais de escolaridade representaram 43 casos, 4,3\% do total. Pacientes sem nenhuma escolaridade foram $24 \%$ do total, com 235 óbitos. Com escolaridade de 1 a 3 anos, foram 186 óbitos, 19\% do total. Já entre os pacientes com 4 a 7 anos de escolaridade a taxa de óbito foi de 25,63\% do total, com 251 casos. Por fim, entre os pacientes com escolaridade de 8 a 11 anos houve 161 óbitos, com taxa de 16,44\% do total de 979 casos de óbitos em Sergipe.

O estudo mostrou que ainda falta completa alimentação do sistema, tanto na notificação adequada do número de casos como também no preenchimento epidemiológico completo dos pacientes acometidos. A importância e contribuição de bases de dados como o DATASUS, através de uma maior disponibilidade de informações são essenciais para execução de medidas de ação em saúde direcionadas de acordo com as características epidemiológicas das enfermidades estudadas.

Gráfico 8 - Óbitos segundo a escolaridade.

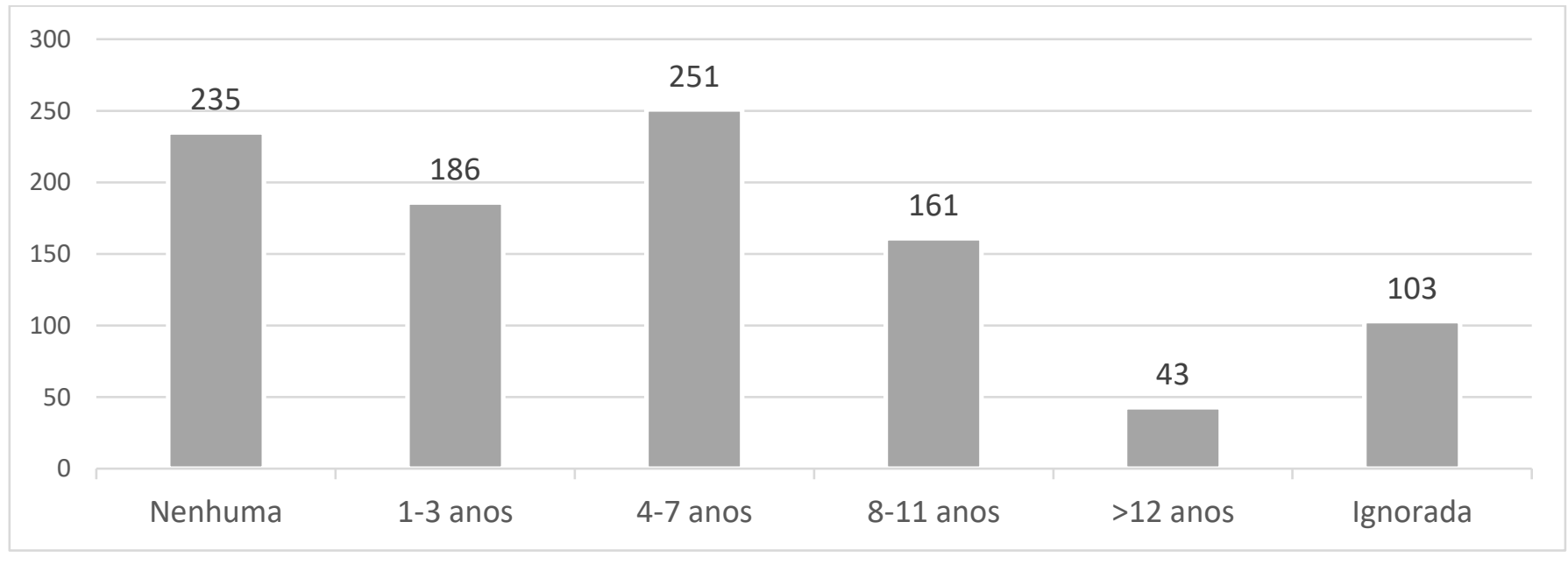

Fonte: Brasil. Ministério da Saúde. Sistema de Informações sobre Mortalidade (SIM). 


\section{Conclusão}

Através desse estudo, foi possível verificar a falta de alimentação do Sistema de Informações Hospitalares do SUS (SIH/SUS) em Sergipe para a doença de Alzheimer, com apenas 21 internações registradas entre 2008 e 2018. Os dados de internamentos hospitalares são mecanismos bastante efetivos que contribuem na tomada de ações das políticas públicas de saúde. Através desses dados epidemiológicos é possível traçar planos de assistência em saúde de acordo com a realidade da população mais acometida. Logo, é preciso verificar o motivo que levou a essa subnotificação do sistema com o objetivo de redimensionar as medidas efetivas na assistência da saúde pública contra as doenças neurodegenerativas.

Ao relacionar os dados encontrados nesta pesquisa com outros estudos internacionais e realizados no Brasil sobre mortalidade da Doença de Alzheimer, realizados em períodos distintos, foi possível observar uma variação de resultados. Por não ser um estudo prospectivo, não houve possibilidade de avaliar se alguns dos dados descritos podem ser confirmados como fator de risco para mortalidade na DA. O sexo feminino representou a maior parte dos óbitos registrados, mas não foi possível avaliar se foi devido ao maior número de diagnóstico nessa população ou ratifica estudos realizados no Brasil em contrapartida aos estrangeiros que descrevem o sexo masculino como fator de risco para mortalidade na DA.

No período avaliado foram registrados 979 óbitos no Estado de Sergipe segundo o Sistema de Informações sobre Mortalidade (SIM) do Ministério da Saúde. Houve uma ascensão do número de óbitos no período avaliado. A faixa etária de 80 anos ou mais foi a mais acometida. Esses dados são resultados da associação de dois fatores: envelhecimento populacional brasileiro, devido à maior expectativa de vida assim como um maior número de diagnóstico da Doença de Alzheimer, proporcionado pelo maior acesso da população à saúde, com esses indivíduos sendo os acometidos pela progressão da doença e mais afetados pelas complicações do estágio final da DA.

A maioria dos óbitos ocorreu em hospitais, decorrente de complicações do estágio final da doença, como broncoaspiração levando a pneumonia, alterações cardiovasculares, distúrbios de deglutição assim como os efeitos adversos devido ao uso de múltiplas medicações. Um fator já descrito como protetor para mortalidade em diversos estudos e ratificado através desse estudo foi o nível de escolaridade. Os pacientes com 12 anos ou mais de escolaridade foram os menos afetados.

A importância e contribuição de bases de dados como o DATASUS, através de uma maior disponibilidade de informações são essenciais para execução de medidas de ação em saúde direcionadas de acordo com as características epidemiológicas das enfermidades estudadas. Portanto, é necessário um melhor registro da história dos pacientes admitidos buscando comorbidades que possam ter contribuído para as afecções presentes no momento da admissão.

Para trabalhos futuros, sugere-se uma investigação aprofundada dos casos de internamento hospitalar, com principais comorbidades associadas ao quadro assim como um melhor detalhamento da causa mortis no paciente com Alzheimer.

\section{Referências}

Agnihotri, A., \& Aruoma, O. I. (2020). Alzheimer's Disease and Parkinson's Disease: A Nutritional Toxicology Perspective of the Impact of Oxidative Stress, Mitochondrial Dysfunction, Nutrigenomics and Environmental Chemicals. Journal of the American College of Nutrition, 39(1), 16-27.

Alzheimer's Disease International. (2019). World Alzheimer Report 2019: Attitudes to dementia. Alzheimer's Disease International (ADI).

Andrews, S. J., Fulton-Howard, B., \& Goate, A. (2020). Interpretation of risk loci from genome-wide association studies of Alzheimer's disease. Lancet Neurology, 19(4), 326-335.

Barrett, B., Schultz, S. K., Luther, S. L., \& Friedman, Y. (2020). Mortality and Associated Risk Factors in Community-Dwelling Persons With Early Dementia. Alzheimer Disease \& Associated Disorders, 34(1), 40-46.

Bellenguez, C., Grenier-Boley, B., \& Lambert, J.-C. (2020). Genetics of Alzheimer's disease: where we are, and where we are going. Current Opinion in Neurobiology, 61, 40-48.

Benwell, C. S., Davila-Pérez, P., Fried, P. J., Jones, R. N., Travison, T. G., Santarnecchi, E., \& Shafi, M. M. (2020). EEG spectral power abnormalities and their relationship with cognitive dysfunction in patients with Alzheimer's disease and Type 2 Diabetes. Neurobiology of Aging, 85, 83-95.

Brasil. IBGE. (2020). Censo Demográfico 2010. Fonte: http://tabnet.datasus.gov.br/cgi/tabcgi.exe?ibge/cnv/popse.def 
Brasil. Ministério da Saúde. (11 de Novembro de 2020). Sistema de Informações sobre Mortalidade (SIM). Fonte: http://tabnet.datasus.gov.br/cgi/tabcgi.exe?sim/cnv/obt10se.def

Brasil. Ministério da Saúde. (11 de Novembro de 2020). Sistema de Informações Hospitalares do SUS (SIH/SUS). Fonte: http://tabnet.datasus.gov.br/cgi/tabcgi.exe?sih/cnv/nrSE.def

Briels, C., Stam, C., Scheltens, P., Bruins, S., Lues, I., \& Gouw, A. (2020). In pursuit of a sensitive EEG functional connectivity outcome measure for clinical trials in Alzheimer's disease. Clinical Neurophysiology, 131(1), 88-95.

CAI, Q., \& Jeong, Y. Y. (2020). Mitophagy in Alzheimer’s Disease and Other Age-Related Neurodegenerative Diseases. Cells, 9(150), 1-28.

Carneiro, P., Morais, S., \& Pereira, M. d. (2020). Biosensors on the road to early diagnostic and surveillance of Alzheimer's disease. Talanta, 211.

Cheng, A., Wang, J., Ghena, N., Zhao, Q., Perone, I., King, T. M., \& Mattson, M. P. (2020). SIRT3 Haploinsufficiency Aggravates Loss of GABAergic Interneurons and Neuronal Network Hyperexcitability in an Alzheimer's Disease Model. Journal of Neuroscience, 40(3), 694-709.

Esperón, J. M. (2017). Pesquisa Quantitativa na Ciência da Enfermagem. Escola Anna Nery.

Franzmeier, N., Neitzel, J., Rubinski, A., Smith, R., Strandberg, O., \& Ossenkoppele, R. (2020). Functional brain architecture is associated with the rate of tau accumulation in Alzheimer's disease. Nature Communication, 11(347).

GATE, D., Saligrama, N., Leventhal, O., YANG, A. C., Unger, M. S., \& Middeldorp, J. (2020). Clonally expanded CD8 T cells patrol the cerebrospinal fluid in Alzheimer's disease. Nature, 577, 399-404.

GBD 2016 Dementia Collaborators. (2019). Global, regional, and national burden of Alzheimer's disease and other dementias, 1990-2016: a systematic analysis for the Global Burden of Disease Study 2016. Lancet Neurology, 18(1), 88-106.

Gil, A. C. (2002). Como Elaborar Projetos de Pesquisa (4a ed.). Atlas.

Hao, X., Bao, Y., Guo, Y., Yu, M., Zhang, D., Risacher, S. L., \& Shen., L. (2020). Multi-modal neuroimaging feature selection with consistent metric constraint for diagnosis of Alzheimer's disease. Medical Image Analysis, 60.

Heneka, M. T. (2020). T cells make a home in the degenerating brain. Nature, 577, 322-323.

Hochman, B., Nahas, F. X., Filho, R. S., \& Ferreira, L. M. (2005). Desenhos de pesquisa. Acta Cirúrgica Brasileira.

Janelidze, S., Mattsson, N., Palmqvist, S., \& Smith, R. (2020). Plasma P-tau181 in Alzheimer's disease: relationship to other biomarkers, differential diagnosis, neuropathology and longitudinal progression to Alzheimer's dementia. Nature Medicine, 26, 379-386.

Jia, L., Quan, M., Fu, Y., Zhao, T., Li, Y., Wei, C., \& Wang, Y.-J. (2020). Dementia in China: epidemiology, clinical management, and research advances. Lancet Neurology, 19(1), 81-92.

Karikari, T. K., Pascoal, T. A., Ashton, N. J., Janelidze, S., Benedet, A. L., Rodriguez, J. L., \& Savard, M. (2020). Blood Phosphorylated Tau 181 as a Biomarker for Alzheimer's Disease: A Diagnostic Performance and Prediction Modelling Study Using Data From Four Prospective Cohorts. Lancet Neurology, 19(5), 422433.

Lima-Costa, M. F., \& Barreto, S. M. (2003). Tipos de estudos epidemiológicos: conceitos básicos e aplicações na área do envelhecimento. Epidemiologia e Serviços de Saúde.

Loureiro, J. C., Pais, M. V., Stella, F., Radanovic, M., \& Teixeira, A. L. (2020). Passive antiamyloid immunotherapy for Alzheimer's disease. Current Opinion in Psychiatry, 33(3), 284-291.

Möllers, T., Perna, L., Stocker, H., Ihle, P., Schubert, I., \& B. S. (2020). Alzheimer's disease medication and outcomes of hospitalisation among patients with dementia. Epidemiology and Psychiatric Sciences, 29(73), 1-9.

Pais, M., Martinez, L., Ribeiro, O., Loureiro, J., Fernandez, R., \& Valiengo, L. (2020). Early diagnosis and treatment of Alzheimer’s disease:. Revista Brasileira de Psiquiatria.

Pini, L., Geroldi, C., Galluzzi, S., Baruzzi, R., Bertocchi, M., \& Chitò, E. (2020). Age at onset reveals different functional connectivity abnormalities in prodromal Alzheimer's disease. Brain Imaging and Behavior.

Piovezan, R. D., Oliveira, D., Arias, N., Acosta, D., \& Prince, M. J. (2020). Mortality Rates and Mortality Risk Factors in Older Adults with Dementia from Low- and Middle-Income Countries: The 10/66 Dementia Research Group Population-Based Cohort Study. Journal of Alzheimer's Disease, 75, $581-593$.

Rubenstein, E., Hartley, S., \& Bishop, L. (2020). Epidemiology of Dementia and Alzheimer Disease in Individuals With Down Syndrome. JAMA Neurology, $77(2), 262-264$.

Santos, C. d., Bessa, T. A., \& Xavier, A. J. (2020). Fatores associados à demência em idosos. Ciência e Saúde Coletiva, 25(2).

Savulich, G., O’Brien, J. T., \& Sahakian, B. J. (2020). Are neuropsychiatric symptoms modifiable risk factors for cognitive decline in Alzheimer's disease and vascular dementia? The British Journal of Psychiatry, 216(1), 1-3.

Scheltens, P., Blennow, K., Breteler, M. M., Strooper, B. d., Frisoni, G. B., Salloway, S., \& Flier, W. M. (2016). Alzheimer’s disease. Lancet, $388,505-517$.

Souza, R. K., Barboza, A. F., Gasperin, G., Garcia, H. D., Barcellos, P. M., \& Nisihara, R. (2020). Prevalência de demência em pacientes atendidos em um hospital privado no sul do Brasil. Einstein, 18, 1-7. 
Research, Society and Development, v. 10, n. 4, e50010414391, 2021

(CC BY 4.0) | ISSN 2525-3409 | DOI: http://dx.doi.org/10.33448/rsd-v10i4.14391

Suryadevara, V., Klüppel, M., Monte, F. d., \& Willis, M. S. (2020). The Unraveling: Cardiac and Musculoskeletal Defects and Their Role in Common Alzheimer Disease Morbidity and Mortality. The American Journal of Pathology, 190(8), 1609-1621.

Webers, A., Heneka, M. T., \& Gleeson, P. A. (2019). The role of innate immune responses and neuroinflammation in amyloid accumulation and progression of Alzheimer's disease. Immunology \& Cell Biology, 98(1), 28-41.

Wen, J., Thibeau-Sutre, E., Routier, A., \& Diaz-Melo, M. (2020). Convolutional neural networks for classification of Alzheimer's disease: Overview and reproducible evaluation. Medical Image Analysis, 63, 48.

Williams, D. M., Finan, C., Schmidt, A. F., Burgess, S., \& Hingorani, A. D. (2020). Lipid Lowering and Alzheimer Disease Risk: A Mendelian Randomization Study. Annals of Neurology, 87(1), 30-39.

Zendehbad, A. S., Noroozianb, M., Shakibac, A., Kargard, A., \& Davoudkhanie, M. (2020). Validation of Iranian Smell Identification Test for screening of mild cognitive impairment and Alzheimer's disease. Applied Neuropsychology: Adult, 1-6.

Zhou, Y., Song, W. M., Andhey, P. S., Swain, A., Levy, T., Miller, K. R., \& Grover, S. (2020). Human and mouse single-nucleus transcriptomics reveal TREM2dependent and TREM2-independent cellular responses in Alzheimer's disease. Nature Medicine, 26, 131-142. 